\title{
Norepinephrine Versus Phenylephrine Infusion for Preventing Postspinal Hypotension During Cesarean Section for Twin Pregnancy: A Double- Blinded Randomized Controlled Clinical Trial
}

\section{Zijun Chen \\ West China Second University Hospital of Sichuan University \\ Jieshu Zhou \\ West China Second University Hospital of Sichuan University \\ Li Wan \\ West China Second University Hospital of Sichuan University \\ Han Huang ( $\nabla$ han.huang@scu.edu.cn ) \\ West China Second University Hospital of Sichuan University}

\section{Research Article}

Keywords: Norepinephrine , phenylephrine infusion, postspinal hypotension , cesarean section , twin pregnancy

Posted Date: October 22nd, 2021

DOI: https://doi.org/10.21203/rs.3.rs-955130/v1

License: (c) (i) This work is licensed under a Creative Commons Attribution 4.0 International License. Read Full License

Version of Record: A version of this preprint was published at BMC Anesthesiology on January 8th, 2022. See the published version at https://doi.org/10.1186/s12871-022-01562-3. 


\section{Abstract}

\section{Background}

Compared with singleton pregnancy, twin gestation is featured by a greater increase in cardiac output. Therefore, norepinephrine might be more suitable than phenylephrine for maintaining blood pressure during cesarean section for twins, as phenylephrine causes reflex bradycardia and resultant a decrease in cardiac output. This study was to determine whether norepinephrine was superior to phenylephrine in maintaining maternal hemodynamics during cesarean section for twins.

\section{Methods}

Informed consent was obtained from all the patients before enrollment. In this double-blinded, randomized clinical trial, 100 parturients with twin gestation undergoing cesarean section with spinal anesthesia were randomized to receive prophylactic norepinephrine $(3.2 \mu \mathrm{g} / \mathrm{min})$ or phenylephrine infusion $(40 \mu \mathrm{g} / \mathrm{min})$. The primary outcome was the change of heart rate and blood pressure during the study period. The secondary outcomes were to compare maternal complications, neonatal outcomes, Apgar scores and umbilical blood acid-base status between the two vasopressors.

\section{Results:}

There was no significant difference was observed for the change of heart rate between two vasopressors. The mean standardized area under the curve of heart rate was $78 \pm 12$ with norepinephrine vs. $74 \pm 11$ beats/min with phenylephrine (mean difference $4.4,95 \% \mathrm{Cl}-0.1$ to $9.0 ; P=.0567$ ). The mean standardized area under the curve of systolic blood pressure (SBP) was significantly lower in parturients with norepinephrine, as the mean of differences in standardized AUC of SBP is $6 \mathrm{mmHg}$, with a $95 \% \mathrm{Cl}$ from 2 to $9 \mathrm{mmHg}(P=.0013)$. However, requirements of physician interventions for correcting maternal hemodynamical abnormalities (temporary cessation of vasopressor infusion for reactive hypertension, rescuing vasopressor bolus for hypotension and atropine for heart rate less $<50$ beats/min) and neonatal outcomes were also not significantly different between two vasopressors.

Conclusion: Infusion of norepinephrine was not associated with less decrease in heart rate during cesarean section for twins, compared with phenylephrine.

Clinical trial number: Chinese Clinical Trial Registry (ChiCTR1900021281)

Clinical trial registry URL: http://www.chictr.org.cn/showproj.aspx?proj=35234

\section{Background}


Compared those with singleton pregnancy, the intensified cardiovascular adaptations with twin pregnancy are characterized by more profound increase in cardiac output (CO) starting from the midtrimester [1, 2]. As more than $70 \%$ of the twin pregnancies were delivered by cesarean section $[3,4]$, a stable peri-cesarean maternal hemodynamics with well-maintained $\mathrm{CO}$ is of vital importance for improving both maternal and neonatal outcomes. Currently, anesthetic management of cesarean section with twins is largely the same as with singleton pregnancy, i.e. spinal anesthesia is preferred over general anesthesia and postspinal hypotension should be actively managed with vasopressor.

Phenylephrine is the recommended vasopressor of choice for treating/preventing spinal anesthesia induced hypotension during cesarean section [5]. As a pure $\boldsymbol{\alpha}$-agonist, phenylephrine causes reflex bradycardia which may lead to decrease in maternal CO [6]. Over the past a few years, norepinephrine, a potent $\boldsymbol{\alpha}$-agonist with additional weak $\boldsymbol{\beta}$-adrenergic agonist activity, has been introduced into the practice of obstetric anesthesia . Previous study has suggested that the positive chronotropic action of norepinephrine counteracts reflex bradycardia caused by pure $\boldsymbol{\alpha}$-adrenergic activation and norepinephrine is therefore associated with greater maternal $\mathrm{CO}$ during cesarean section for singleton pregnancy [7]. From this point of view, norepinephrine seems to be the vasopressor of choice for postspinal hypotension during cesarean section with twins. However, there is limited study comparing different vasopressors in setting of twin pregnancy, which were excluded in most, if not all, of the previous studies [7-10].

The primary objective of this randomized, double-blinded study was to compare the changes in heart rate and blood pressure following prophylactic intravenous infusions of norepinephrine or phenylephrine in women with twin pregnancies undergoing cesarean section with spinal anesthesia. The secondary objectives were to maternal complications, neonatal outcomes, Apgar scores and umbilical blood acidbase status between the two vasopressors.

\section{Methods}

This study was reviewed and approved by ethics committee (full name of the committee: China Ethics Committee of Registering Clinical Trials; reference No. ChiECRCT-20190007). Study protocol was registered in the Chinese Clinical Trial Registry prior to patient enrollment (Registration no. ChiCTR1900021281, Principal investigator: Dr. Jieshu Zhou, Date of registration: 11 February 2019, weblink for the online registration: http://www.chictr.org.cn/showproj.aspx?proj=35234). Informed consent was obtained from all patients before enrollment. This manuscript adheres to the applicable CONSORT guidelines (figure 1). The authors assert that all procedures contributing to this work comply with the ethical standards of the relevant national and institutional committees on human experimentation and with the Helsinki Declaration of 1975, as revised in 2008.

Parturients with twin pregnancies were screened according to following inclusion criteria: American Society of Anesthesiologists physical status I-III, gestational age between 32 and $38^{+6}$ weeks, going to receive cesarean section with spinal anesthesia, and estimated body weight $\geq 1500 \mathrm{~g}$ for both fetuses. 
Parturients with cardiovascular disease, hypertensive disorder, onset of labor, contraindications to spinal anesthesia or known severe fetal abnormality would be excluded. Patients who underwent cesarean section due to non-reassuring fetal status or received general anesthesia for the cesarean section would also be excluded.

Upon entering the operating room, patients were positioned on the operating table in supine position tilted to the left for $15^{\circ}$ to avoid possible aortocaval compression as per our department protocol. Patients were also positioned in this left tilt position during the cesarean procedure. Standard monitoring was applied, including 5-lead electrocardiography, non-invasive blood pressure, and pulse oximetry. Blood pressure and heart rate were measured and recorded at 1-min interval till the difference between 3 consecutive measurements was less than 10\%. The mean values of these 3 measurements were recorded as baseline values.

Two 18-G intravenous catheters were inserted into forearm veins while no fluid preload was given. Then, patients were positioned in left lateral position while spinal anesthesia was performed. $2.5 \mathrm{ml}$ of $0.5 \%$ plain isobaric bupivacaine were injected intrathecally at L3-L4 or L2-L3 intervertebral space via a 25-G pencil-point spinal needle. Intrathecal administration of opioids was spared in this study.

Sensory blockade level was assessed by pinprick and surgery would not begin unless the sensory block reached T6 level. Patients with inadequate sensory blockade would receive general anesthesia for cesarean delivery, who would then be excluded from this study.

Infusion of study drug was initiated, when spinal injection started. According to a computer-generated random number table, sequentially numbered opaque sealed envelopes were prepared by one of the investigators (Z.C.). The envelop was opened before spinal anesthesia. Following the instruction of the envelop, the syringe was prepared by J.Z. and labelled as "study drug", which containing either $8 \mu \mathrm{g} / \mathrm{ml}$ norepinephrine or $100 \mu \mathrm{g} / \mathrm{ml}$ phenylephrine. The infusion rate was $24 \mathrm{ml} /$ hour for both vasopressors, corresponding to $3.2 \mu \mathrm{g} / \mathrm{min}$ for norepinephrine and $40 \mu \mathrm{g} / \mathrm{min}$ for phenylephrine. Phenylephrine infusion at $40 \mu \mathrm{g} / \mathrm{min}$ for is our standard protocol. The infusion rate of norepinephrine was then determined based on the estimated potency of 11.3 [11] to 13.1:1 [12] for norepinephrine: phenylephrine. Study drug was flushed into the blood stream by carrier lactated Ringer's solution infused at the rate of $1 \mathrm{ml}^{\prime} \mathrm{kg}^{-1}$. hour $^{-1}$. Infusion of study drug was stopped when the second twin was delivered, and the study was then terminated. Both the patients and the anesthesiologists were blinded for the patients' assignments. Ringer's solution co-loading via another peripheral venous catheter was initiated as infusion of the study drug began. The infusion bags were suspended about 1.5 meter above the parturient's right atrium with the clamp fully opened till the study ended. Then, the fluid infusion rate was adjusted at the discretion of the anesthesiologist.

Blood pressure was measured and recorded every minute till delivery of the second twin. Hypotension, defined as the systolic blood pressure (SBP) $<90 \mathrm{mmHg}$ or $80 \%$ of the baseline SBP, was rescued by $1-\mathrm{ml}$ bolus of the study drug, i.e. norepinephrine $8 \mu \mathrm{g}$ for parturients with norepinephrine infusion and 
phenylephrine $100 \mu \mathrm{g}$ for parturients with phenylephrine. These boluses were prepared beforehand in a $10-\mathrm{ml}$ syringe labelled as "rescuing drug". Hypertension, defined as SBP $>140 \mathrm{mmHg}$ or $120 \%$ of the baseline, was managed by temporarily stopping study drug infusion, which would be restarted as soon as SBP was within the normal range. Heart rate was monitored continuously and recorded at 1-min intervals. Bradycardia was defined as heart rate $<60$ beats $/ \mathrm{min}$ and $0.5 \mathrm{mg}$ atropine would be given when heart rate further decreased below 50 beats/min. Oxygen $5 \mathrm{~L} / \mathrm{min}$ was given by a facemask during the study period.

Apgar scores were assessed by midwives 1, 5 and 10 minutes after delivery, who were unaware of the patients' assignment. Umbilical arterial (UA) and venous blood (UV) were sampled from a doubleclamped segment of umbilical cord by using Pulset arterial $3 \mathrm{cc}$ blood gas syringes (Westmed, AZ85706, USA). Within 20 minutes after clamping, umbilical blood gas was analyzed with the bedside epoc® Blood Analysis System (Siemens Healthcare $\mathrm{GmbH}$, Erlangen, Germany) in the operating theatre.

The primary outcome was the change in heart rate and blood pressure during the study period, from intrathecal injection to delivery of the second twin, which was calculated as the standard area under the curve of heart rate (please find below, in the section of statistical analysis). The secondary outcomes included the incidences of hypotension, hypertension, bradycardia, nausea and vomiting, umbilical blood gas status, Apgar scores, both maternal and neonatal length of post-operative stays, and rates of maternal and neonatal intensive care unit (NICU) admission.

\section{Statistical analysis}

Continuous data such as $\mathrm{pH}$ values were assessed for normal distribution using Kolmogonov-Smirnov test and were then compared using the Student's t-test or Mann-Whitney U test, as appropriate. Categorical data such as the incidence of bradycardia were analyzed using chi-square test. A survival analysis with log-rank test was used to compare the requirement of physician interventions for correcting maternal hemodynamic abnormalities (including injection of rescuing bolus of vasopressors or atropine and temporary cessation of vasopressor infusion). The area under the curves (AUC) for SBP and heart rate plotted against time were calculated using the trapezium rule. As the surgical time varied among parturients, a variable number for heart rate and blood pressure measurements were recorded for each parturient. Each AUC value was divided by the number of data points recorded to give the standardized AUC for each patient, which were compared between two vasopressors using the Student's t-test. Analysis was performed with Prism (version 8.4.3, GraphPad Software, San Diego, California USA). A $p$ value < 0.05 was considered statistically significant.

The sample size was calculated based on our unpublished preliminary data, in which parturients with twin pregnancy received norepinephrine or phenylephrine infusion following spinal anesthesia. The means of standardized AUC for heart rate were $79 \pm 12$ and $72 \pm 8$ beats/min for norepinephrine and phenylephrine, respectively. A minimal sample size of 46 per group was required to detect a significant difference ( $10 \%$ of the baseline heart rate) between two groups, with $90 \%$ power and a two-sided type I 
error of 0.05 . Considering the potential withdraw of consent or loss of follow-up, a final sample size of 50 per group was determined.

\section{Results}

This study was conducted in West China Second University Hospital, Chengdu, China, from November 2019 to April 2020. The CONSORT recruitment diagram is showed in Figure 1. Totally 141 parturients with twin pregnancy were screened for eligibility and data were analyzed from 50 patients in each group.

Patients baseline and surgical characteristics were similar between two groups (Table 1). Infusion of norepinephrine did not lead to less decrease in heart rate following spinal anesthesia in patients with twin pregnancy and there was no inter-group difference in heart rate over the study period (figure 2A). The mean standardized AUC of heart rate was $78 \pm 12$ and $74 \pm 11$ beats/min with norepinephrine and phenylephrine, respectively (mean of difference 4.4 with a $95 \%$ confidence interval $(\mathrm{Cl})-0.1$ to $9.0 ; P=$ .0567). The incidence of bradycardia, i.e. heart rate $<60$ beats/min, was $6 \%(3 / 50)$ in patients receiving norepinephrine and $30 \%(15 / 50)$ in patients with phenylephrine (odds ratios [OR] with norepinephrine = $0.15 ; 95 \% \mathrm{Cl}, 0.04$ to $0.56 ; P=.002$ ), as showed in Table 2 . Among these patients with bradycardia, only three from group phenylephrine met the criteria for atropine administration, i.e. heart rate $<50$ beats $/ \mathrm{min}$ $(3 / 50,6 \%$ versus $0 / 50,0 \%$, OR with norepinephrine $=0 ; 95 \% \mathrm{Cl}, 0$ to $1.11 ; P=.242)$. It is worth further mentioning that $86.7 \%(13 / 15)$ of the bradycardia episodes in group phenylephrine occurred before surgical skin preparation followed by a rapid and spontaneous recovery and only two patients had heart rate less than 60 beats/min when the neonates were delivered. 
Table 1

Baseline and Surgical Characteristics

\begin{tabular}{|c|c|c|c|}
\hline & $\begin{array}{l}\text { NE } \\
(n=50)\end{array}$ & $\begin{array}{l}\mathrm{PE} \\
(\mathrm{n}=50)\end{array}$ & $P$ value \\
\hline Age, year & $31.6(4.3)$ & $31.5(4.2)$ & .400 \\
\hline Weight, kg & $72.6(8.0)$ & $71.0(11.2)$ & .415 \\
\hline Height, cm & $161(5.3)$ & $160(4.7)$ & .464 \\
\hline Body mass index, $\mathrm{kg} \cdot \mathrm{m}^{-2}$ & $28.0(2.3)$ & $27.6(3.5)$ & .505 \\
\hline Gestation at delivery, week & & & .913 \\
\hline $32^{+0}$ to $34^{+6}$ & $8(16 \%)$ & $7(14 \%)$ & \\
\hline $35^{+0}$ to $36^{+6}$ & $21(42 \%)$ & $23(46 \%)$ & \\
\hline $37^{+0}$ to $38^{+6}$ & $21(42 \%)$ & $20(40 \%)$ & \\
\hline Parity & $1(0-2)$ & $1(0-2)$ & .437 \\
\hline Pregnancy complications ${ }^{a}$ & $23(46 \%)$ & $18(36 \%)$ & .309 \\
\hline Block height, dermatome & $6(4-6)$ & $6(4-6)$ & .060 \\
\hline Baseline systolic blood pressure, $\mathrm{mmHg}$ & $116(10)$ & $118(8)$ & .250 \\
\hline Baseline heart rate, beats/min & $89(12)$ & $87(12)$ & .419 \\
\hline \multicolumn{4}{|l|}{ Time intervals, second } \\
\hline Spinal injection to skin incision & $1032(877-1215)$ & $1069(750-1261)$ & .907 \\
\hline Skin incision to delivery of first twin & $247(204-345)$ & $230(184-306)$ & .272 \\
\hline Interval between two deliveries & $72(54-108)$ & $63(43-105)$ & .244 \\
\hline Blood Loss, ml & $539(156)$ & $551(203)$ & .741 \\
\hline Nausea and vomiting & $2(4 \%)$ & $1(2 \%)$ & 1.000 \\
\hline Postoperative length of stay, day & $3(3-4)$ & $3(3-4)$ & .917 \\
\hline \multicolumn{4}{|c|}{ Data reported as median mean (standard deviation), (interquartile range), or number (proportion). } \\
\hline \multicolumn{4}{|c|}{$\begin{array}{l}\text { a Pregnancy complications include hypothyroidism, intrahepatic cholestasis of pregnancy and } \\
\text { gestational diabetes. }\end{array}$} \\
\hline \multicolumn{4}{|c|}{ Abbreviations: NE, norepinephrine; $\mathrm{PE}$, phenylephrine. } \\
\hline
\end{tabular}


Table 2

Incidence of Maternal Hemodynamic Abnormalities

\begin{tabular}{|c|c|c|c|c|}
\hline & $\begin{array}{l}\text { NE } \\
(n=50)\end{array}$ & $\begin{array}{l}\text { PE } \\
(n=50)\end{array}$ & OR with NE & $P$ value \\
\hline Bradycardia $^{a}$ & $3(6 \%)$ & $15(30 \%)$ & $0.15(0.04,0.56)$ & .002 \\
\hline Atropine rescue ${ }^{b}$ & $0(0 \%)$ & $3(6 \%)$ & $0.00(0.00,1.11)$ & .242 \\
\hline Hypotension & $12(24 \%)$ & $3(6 \%)$ & $4.95(1.30,18.81)$ & .012 \\
\hline Hypertension & $6(12 \%)$ & $13(26 \%)$ & $0.39(0.13,1.12)$ & .074 \\
\hline \multicolumn{5}{|c|}{ Data reported as number (proportion) or OR [95\% Cl]. } \\
\hline \multicolumn{5}{|c|}{${ }^{a}$ Bradycardia is defined as heart rate is less than 60 beats $/ \mathrm{min}$. } \\
\hline \multicolumn{5}{|c|}{${ }^{b}$ Atropine is administrated when heart rate is less than 50 beats $/ \mathrm{min}$. } \\
\hline
\end{tabular}

Patients receiving norepinephrine had significantly lower blood pressure than those receiving phenylephrine during the study period, as revealed in figure 2B. However, the mean of differences in standardized AUC of SBP is $6 \mathrm{mmHg}$, with a $95 \% \mathrm{Cl}$ from 2 to $9 \mathrm{mmHg}(P=.0013)$. The incidence of hypotension was $24 \%(12 / 50)$ with norepinephrine and $6 \%(3 / 50)$ with phenylephrine (OR with norepinephrine $=4.95 ; 95 \% \mathrm{Cl}, 1.30-18.81 ; P=.012$ ). None of the hypotensive patients required more than one bolus of rescuing vasopressor to restore blood pressure. As rescuing bolus was used more frequently in patients with norepinephrine, the total volume of vasopressor was greater in group norepinephrine: 9.9 \pm 2.3 versus $8.5 \pm 2.9 \mathrm{~mL}$ with mean difference of $1.45 ; 95 \% \mathrm{Cl}, 0.42$ to $2.48(P=.006)$. Hypertension occurred in 6 patients receiving norepinephrine $(12 \%, 6 / 50)$ and in 13 patients receiving phenylephrine $(26 \%, 13 / 50)$, OR with norepinephrine $=0.39 ; 95 \% \mathrm{Cl}, 0.13-1.12,(P=.074)$. Taking all the abovementioned maternal hemodynamic abnormalities into consideration (including atropine for heart rate < 50 beats/min, bolus of rescuing vasopressors for hypotension and temporary cessation of study drug infusion for reactive hypertension), the requirements of physician intervention were similar between the two groups (figure 3).

No newborn had Apgar's score less than 8 or unplanned NICU admission in either group. As showed in Table 3, there was no difference in neonatal outcomes between the two groups, except that neonates in group norepinephrine had a slightly greater body weight. Due to the equipment failure and difficulties in collecting blood, umbilical arterial (UA) blood gas analysis was missed in 13 and 11 neonates in group norepinephrine and phenylephrine, respectively; umbilical venous (UV) blood gas analysis was missed in 9 neonates in each group. No UV pH value was less than 7.20 in either group while UA pH $<7.20$ was found in 2.3\% (2/87) of newborns from group norepinephrine and $1.1 \%(1 / 89)$ of newborns from group phenylephrine (OR with norepinephrine $=2.05 ; 95 \% \mathrm{Cl}, 0.24-30.31, P=.619)$. Umbilical blood gas status 
was similar between the two groups, except that neonates from group norepinephrine had a slightly higher glucose level, in both UA blood ( $2.92 \pm 0.57$ versus $2.71 \pm 0.56 \mathrm{mmol} / \mathrm{L}, P=.015)$ and UV blood $(3.39 \pm 0.57$ versus $3.18 \pm 0.67 \mathrm{mmol} / \mathrm{L}, P=.020)$. 
Table 3

Neonatal Outcomes Compared between norepinephrine and phenylephrine

\begin{tabular}{|c|c|c|c|c|}
\hline & \multirow{2}{*}{$\begin{array}{l}\text { NE } \\
(n=100)\end{array}$} & \multirow{2}{*}{$\begin{array}{l}\text { PE } \\
(n=100)\end{array}$} & \multirow[t]{2}{*}{$95 \% \mathrm{Cl}^{a}$} & \multirow[t]{2}{*}{$P$ value } \\
\hline & & & & \\
\hline Birthweight, g & $2493(388)$ & $2389(331)$ & $104(3.3,204.5)$ & .043 \\
\hline$<2500$ & $47(47 \%)$ & $60(60 \%)$ & $0.59(0.34,1.04)$ & .065 \\
\hline$<2000$ & $14(14 \%)$ & $10(10 \%)$ & $1.47(0.62,3.48)$ & .384 \\
\hline Height, cm & $46(2.5)$ & $46(2.3)$ & $0.3(-0.37,0.97)$ & .379 \\
\hline Sex & & & $1.33(0.76,2.32)$ & .320 \\
\hline Female & $42(42 \%)$ & 49 (49\%) & & \\
\hline Male & $58(58 \%)$ & $51(51 \%)$ & & \\
\hline \multicolumn{5}{|l|}{ Apgar score } \\
\hline $1 \mathrm{~min}$ & $10(8-10)$ & $10(8-10)$ & $0.00(0.00,0.00)$ & .764 \\
\hline $5 \mathrm{~min}$ & $10(9-10)$ & $10(9-10)$ & $0.00(0.00,0.00)$ & .562 \\
\hline $10 \mathrm{~min}$ & $10(9-10)$ & $10(9-10)$ & $0.00(0.00,0.00)$ & .156 \\
\hline NICU Admission & $27(27 \%)$ & $30(30 \%)$ & $0.86(0.47,1.60)$ & .638 \\
\hline UA blood gas & $\mathrm{n}=87$ & $\mathrm{n}=89$ & & \\
\hline $\mathrm{pH}$ & $7.292(0.036)$ & $7.295(0.035)$ & $-0.003(-0.014,0.007)$ & .538 \\
\hline $\mathrm{PCO}_{2}, \mathrm{mmHg}$ & $48.66(5.39)$ & $48.70(6.38)$ & $-0.03(-1.79,1.72)$ & .969 \\
\hline $\mathrm{PO}_{2}, \mathrm{mmHg}$ & $19.74(4.79)$ & $18.36(5.123)$ & $1.38(-0.10,2.86)$ & .067 \\
\hline $\mathrm{BE}, \mathrm{mmol} / \mathrm{l}$ & $-3.09(1.99)$ & $-2.93(1.91)$ & $-0.17(-0.75,0.41)$ & .57 \\
\hline $\mathrm{SO}_{2}, \%$ & $26.32(10.64)$ & 23.90 (11.97) & $2.42(-0.95,5.79)$ & .158 \\
\hline Glu, mmol/l & $2.92(0.57)$ & $2.71(0.56)$ & $0.21(0.04,0.38)$ & .015 \\
\hline Lac, mmol/I & $2.83(0.70)$ & $2.76(0.67)$ & $0.06(-0.14,0.27)$ & .544 \\
\hline UV blood gas & $\mathrm{n}=91$ & $\mathrm{n}=91$ & & \\
\hline
\end{tabular}

Data reported as mean (standard deviation), median (range), or number (proportion).

a $95 \% \mathrm{Cl}$ is $95 \%$ confidence interval of difference or odds ratio.

Abbreviations: BE, base excess; Glu, glucose; Lac, lactate; NE, norepinephrine; NICU, Neonatal intensive care unit; $P E$, phenylephrine; UA Umbilical artery; UV Umbilical vein. 


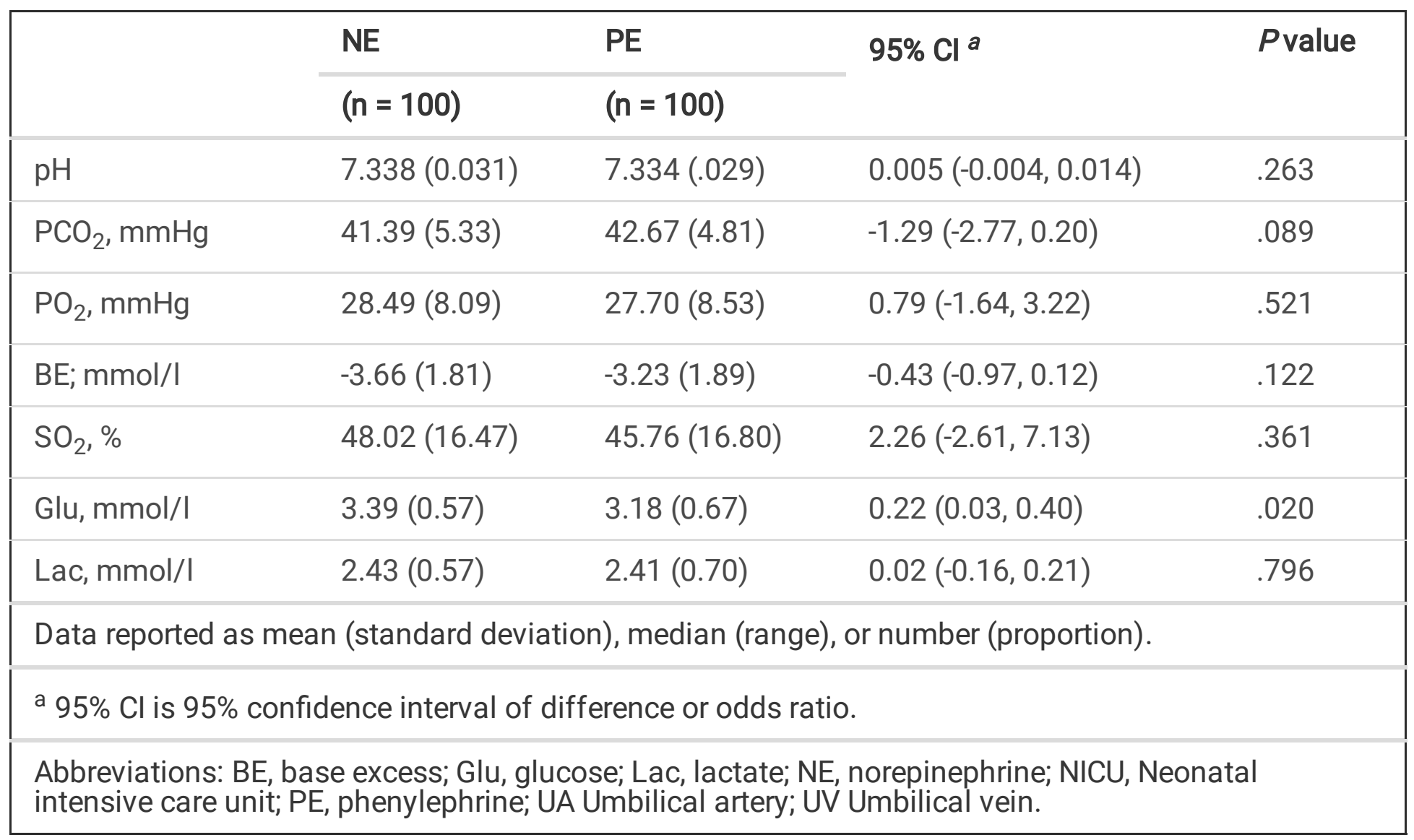

\section{Discussion}

Our study demonstrated that compared with phenylephrine, prophylactic infusion of norepinephrine was not associated with less decrease in heart rate during cesarean delivery for twin pregnancy with spinal anesthesia, but with a higher incidence of hypotension. Overall, requirements of physician interventions to correcting maternal hemodynamic abnormalities, (i.e. atropine for heart rate less than 50 beats $/ \mathrm{min}$, rescuing vasopressor bolus for hypotension and temporary cessation of vasopressor infusion for reactive hypertension), were similar between two vasopressors. There was no difference in fetal outcomes either.

Most of which were delivered by cesarean section [3, 4], and anesthetic management of cesarean section with twins are largely the same as for singleton pregnancy [13]. From this point of view, phenylephrineinduced bradycardia and resultant decrease in cardiac output (CO) [14] seems a particular concern for cesarean section with twin pregnancy, as a higher heart rate contributed substantially to the augmented CO developed in twin pregnancy [2].

Similar to the results from previous study with singleton pregnancy, infusion of norepinephrine was not associated with greater heart rate [10] or greater CO [15], in patients twin pregnancy receiving spinal anesthesia for cesarean delivery. Although the incidence of bradycardia was higher in patients receiving phenylephrine, the requirements of atropine were not different between the two vasopressors. Furthermore, $86.7 \%(13 / 15)$ of the bradycardia episodes occurred before skin incision, with a spontaneous recover afterwards. From the eleventh recording of blood pressure after intrathecal injection, there was no difference in heart rate between the two groups while most of the neonates were delivered 
thereafter. Therefore, transient decrease in heart rate following phenylephrine infusion, as observed in our study, might not be a concern for potentially impaired placental perfusion as the heart rate recovered before delivery of the newborns. Furthermore, it seemed that phenylephrine infusion at $40 \mu \mathrm{g} / \mathrm{min}$ is too high for women with twin pregnancy as more than $20 \%$ of these patients developed reactive hypertension. We speculate that a slower infusion rate would lead to a less suppression of heart rate and a lower incidence of bradycardia.

The infusion rate of norepinephrine was determined based on the estimated potency of 11.3 [11] to 13.1:1 [12] for norepinephrine : phenylephrine, as infusion rate of $40 \mu \mathrm{g} / \mathrm{min}$ for phenylephrine is our established departmental protocol. In our study, infusion of norepinephrine at $3.2 \mu \mathrm{g} / \mathrm{min}$ corresponds to a weight-adjusted infusion rate of $0.044 \mu \mathrm{g} \cdot \mathrm{kg}^{-1} \cdot \mathrm{min}^{-1}$ (ranging from 0.034 to $0.056 \mu \mathrm{g} \cdot \mathrm{kg}^{-1} \cdot \mathrm{min}^{-1}$ ), which is very close to the recommended rate of $0.05 \mu \mathrm{g} \cdot \mathrm{kg}^{-1} \cdot \mathrm{min}^{-1}$ from previous study [16], as a higher infusion rate of $0.075 \mu \mathrm{g} \cdot \mathrm{kg}^{-1} \cdot \mathrm{min}^{-1}$ did not further reduce postspinal anesthesia hypotension during cesarean section. The incidences of hypotension were also very close between these two studies, $24 \%$ in ours versus $24.7 \%$ as reported [16]. However, it is worth noting that in Hasanin's study [16], $5 \mu \mathrm{g}$ norepinephrine bolus was given preceding norepinephrine infusion while we used norepinephrine infusion alone. In other studies with norepinephrine infusion at the rate of $0.05 \mu \mathrm{g} \cdot \mathrm{kg}^{-1} \cdot \mathrm{min}^{-1}$ alone in singleton pregnancy, the incidence of hypotension was as high as 30\% [17] or even $47.4 \%$ [18]. In two recently published paper, which were not available when our study was designed, the $\mathrm{ED}_{95}$ of norepinephrine for maintaining blood pressure for cesarean delivery after spinal anesthesia was estimated to be 0.07 [18] to $0.08[17] \mu \mathrm{g} \cdot \mathrm{kg}^{-1} \cdot \mathrm{min}^{-1}$. However, these data were both derived from singleton pregnancy and were with wide confidence intervals, so it should be cautious to directly extrapolate these data to twin pregnancy. Equipotency of both vasopressors need to be confirmed in women with twin pregnancy and maternal/fetal outcomes should be compared with these equipotent regimens in future studies.

In this study, we included a wide spectrum of gestational ages, which is not uncommon in studies of twin pregnancy [13]. Although gestational age could impact hypotension rates during caesarean delivery, the equal randomization of gestational ages between groups in our study suggest that this had little impact on between group differences (Table 1).

Finally, it is worth noting that the sensory blockade in our study is lower in our study (T6 versus T4), compared with some of the previous studies [7, 9]. This reflects the prevailing clinical practice in our department. We believe that blockade level at T6 would be sufficient for cesarean section as no patient in our study reported any discomfort due to incomplete blockade or required any supplementary intravenous analgesics or anesthetics during the cesarean procedure. Others also reported the same level of blockade for cesarean delivery under spinal anesthesia [10], [17], [18].

There are several limitations in our study. First, vasopressors were infused at a fixed rate for all the patients, instead of a weight-based infusion or closed-loop feedback computer-controlled infusion. Our hospital is a major teaching hospital with a high-volume tertiary obstetric center and time for precesarean preparation is limited. We did not have the manpower to prepare the infusion vasopressor in a 
timely manner and we believed that it is very likely to cause more infusion errors. More importantly, the incidence of hypotension reported in our study is comparable to those reported before [16-18]. Therefore, the relatively simple infusion protocol of our study should not cast any doubt on the validity of our results. Further study is warranted to define the best infusion protocol. Another limitation is that we did not measure maternal cardiac output directly, but measured heart rate which is regarded as the "best surrogate indicator of cardiac output during cesarean delivery" [19]. The major reason for the missing of cardiac output measurement is that there was a hardware failure of our bioimpedance-based noninvasive $\mathrm{CO}$ monitoring device just before the study began, therefore the availability of this equipment cannot be guaranteed for our study. Moreover, there have been concerns about the accuracy of noninvasive CO monitors especially when used in pregnant women [20]. Therefore, the heart rate was considered as a surrogate.

In conclusion, in parturients with twin pregnancy undergoing cesarean delivery under spinal anesthesia, infusion of norepinephrine at the rate of $3.2 \mu \mathrm{g} / \mathrm{min}$ was not associated with less decrease in heart rate, compared with phenylephrine infused at $40 \mu \mathrm{g} / \mathrm{min}$. The requirements of physician intervention for correcting maternal hemodynamical abnormalities and fetal outcomes were also similar between two vasopressors.

\section{Declarations}

\section{Ethics approval and consent to participate}

This study was reviewed and approved by China Ethics Committee of Registering Clinical Trials (Reference No. ChiECRCT-20190007). Study protocol was registered in the Chinese Clinical Trial Registry prior to patient enrollment (Registration no. ChiCTR1900021281, Date of registration: 11 February 2019 and weblink for the online registration: http://www.chictr.org.cn/showproj.aspx? proj=35234). Informed consent was obtained from all patients before enrollment.

\section{Consent for publication}

No individual person's data was included or revealed in this study.

\section{Availability of data and materials}

All data generated or analysed during this study are included in this published article.

\section{Competing interests}

None of the authors declare any competing interests. 


\section{Funding}

Dr. Han Huang received funding support from Chengdu Bureau of Science and Technology (2019-YFYF00108-SN).

\section{Authors' contributions}

ZC and JZ helped in patients' recruitment, data collection, data analysis and drafting the manuscript. LW helped in data collection. HH designed this study, analyzed data and wrote the manuscript.

\section{Acknowledgements}

The authors would like to thank Dr. Rong Zhou and all the staff in our labor ward for their generous support during this study.

\section{References}

1. Kuleva M, Youssef A, Maroni E, Contro E, Pilu G, Rizzo N, Pelusi G, Ghi T: Maternal cardiac function in normal twin pregnancy: a longitudinal study. Ultrasound in obstetrics \& gynecology: the official journal of the International Society of Ultrasound in Obstetrics and Gynecology 2011, 38(5):575-580.

2. Kametas NA, McAuliffe F, Krampl E, Chambers J, Nicolaides KH: Maternal cardiac function in twin pregnancy. Obstetrics and gynecology 2003, 102(4):806-815.

3. Lee HC, Gould JB, Boscardin WJ, El-Sayed YY, Blumenfeld YJ: Trends in cesarean delivery for twin births in the United States: 1995-2008. Obstetrics and gynecology 2011, 118(5):1095-1101.

4. Bateni ZH, Clark SL, Sangi-Haghpeykar H, Aagaard KM, Blumenfeld YJ, Ramin SM, Lee HC, Fox KA, Moaddab A, Shamshirsaz AA et al: Trends in the delivery route of twin pregnancies in the United States, 2006-2013. Eur J Obstet Gynecol Reprod Biol 2016, 205:120-126.

5. Kinsella SM, Carvalho B, Dyer RA, Fernando R, McDonnell N, Mercier FJ, Palanisamy A, Sia ATH, Van de Velde $M$, Vercueil $A$ et al: International consensus statement on the management of hypotension with vasopressors during caesarean section under spinal anaesthesia. Anaesthesia 2018, 73(1):7192.

6. Stewart A, Fernando R, McDonald S, Hignett R, Jones T, Columb M: The dose-dependent effects of phenylephrine for elective cesarean delivery under spinal anesthesia. Anesthesia and Analgesia 2010, 111(5):1230-1237.

7. Ngan Kee WD, Lee SW, Ng FF, Tan PE, Khaw KS: Randomized double-blinded comparison of norepinephrine and phenylephrine for maintenance of blood pressure during spinal anesthesia for cesarean delivery. Anesthesiology 2015, 122(4):736-745. 
8. Sharkey AM, Siddiqui N, Downey K, Ye XY, Guevara J, Carvalho JCA: Comparison of Intermittent Intravenous Boluses of Phenylephrine and Norepinephrine to Prevent and Treat Spinal-Induced Hypotension in Cesarean Deliveries: Randomized Controlled Trial. Anesth Analg 2019, 129(5):13121318.

9. Vallejo MC, Attaallah AF, Elzamzamy OM, Cifarelli DT, Phelps AL, Hobbs GR, Shapiro RE, Ranganathan P: An open-label randomized controlled clinical trial for comparison of continuous phenylephrine versus norepinephrine infusion in prevention of spinal hypotension during cesarean delivery. Int J Obstet Anesth 2017, 29:18-25.

10. Mohta M, Garg A, Chilkoti GT, Malhotra RK: A randomised controlled trial of phenylephrine and noradrenaline boluses for treatment of postspinal hypotension during elective caesarean section. Anaesthesia 2019, 74(7):850-855.

11. Mohta M, Dubey M, Malhotra RK, Tyagi A: Comparison of the potency of phenylephrine and norepinephrine bolus doses used to treat post-spinal hypotension during elective caesarean section. Int J Obstet Anesth 2019, 38:25-31.

12. Ngan Kee WD: A Random-allocation Graded Dose-Response Study of Norepinephrine and Phenylephrine for Treating Hypotension during Spinal Anesthesia for Cesarean Delivery. Anesthesiology 2017, 127(6):934-941.

13. Barrett JF, Hannah ME, Hutton EK, Willan AR, Allen AC, Armson BA, Gafni A, Joseph KS, Mason D, Ohlsson A et al: A randomized trial of planned cesarean or vaginal delivery for twin pregnancy. $N$ Engl J Med 2013, 369(14):1295-1305.

14. Heesen M, Hilber N, Rijs K, Rossaint R, Girard T, Mercier FJ, Klimek M: A systematic review of phenylephrine vs. noradrenaline for the management of hypotension associated with neuraxial anaesthesia in women undergoing caesarean section. Anaesthesia 2020, 75(6):800-808.

15. Feng K, Wang X, Feng X, Zhang J, Xiao W, Wang F, Zhou Q, Wang T: Effects of continuous infusion of phenylephrine vs. norepinephrine on parturients and fetuses under LiDCOrapid monitoring: a randomized, double-blind, placebo-controlled study. BMC Anesthesiol 2020, 20(1):229.

16. Hasanin AM, Amin SM, Agiza NA, Elsayed MK, Refaat S, Hussein HA, Rouk TI, Alrahmany M, Elsayad $\mathrm{ME}$, Elshafaei KA et al: Norepinephrine Infusion for Preventing Postspinal Anesthesia Hypotension during Cesarean Delivery: A Randomized Dose-finding Trial. Anesthesiology 2019, 130(1):55-62.

17. Fu F, Xiao F, Chen W, Yang M, Zhou Y, Ngan Kee WD, Chen X: A randomised double-blind doseresponse study of weight-adjusted infusions of norepinephrine for preventing hypotension during combined spinalepidural anaesthesia for Caesarean delivery. British journal of anaesthesia 2020, 124(3):e108-e114.

18. Wei C, Qian J, Zhang Y, Chang X, Hu H, Xiao F: Prospective, randomised, double-blind, dose-finding study of norepinephrine for preventing spinal-induced hypotension during caesarean delivery under combined spinalepidural anaesthesia. European journal of anaesthesiology 2020, 23.

19. Dyer RA, Reed AR, van Dyk D, Arcache MJ, Hodges O, Lombard CJ, Greenwood J, James MF: Hemodynamic effects of ephedrine, phenylephrine, and the coadministration of phenylephrine with 
oxytocin during spinal anesthesia for elective cesarean delivery. Anesthesiology 2009, 111(4):753765.

20. Thiele RH, Bartels K, Gan TJ: Cardiac output monitoring: a contemporary assessment and review. Crit Care Med 2015, 43(1):177-185.

\section{Figures}

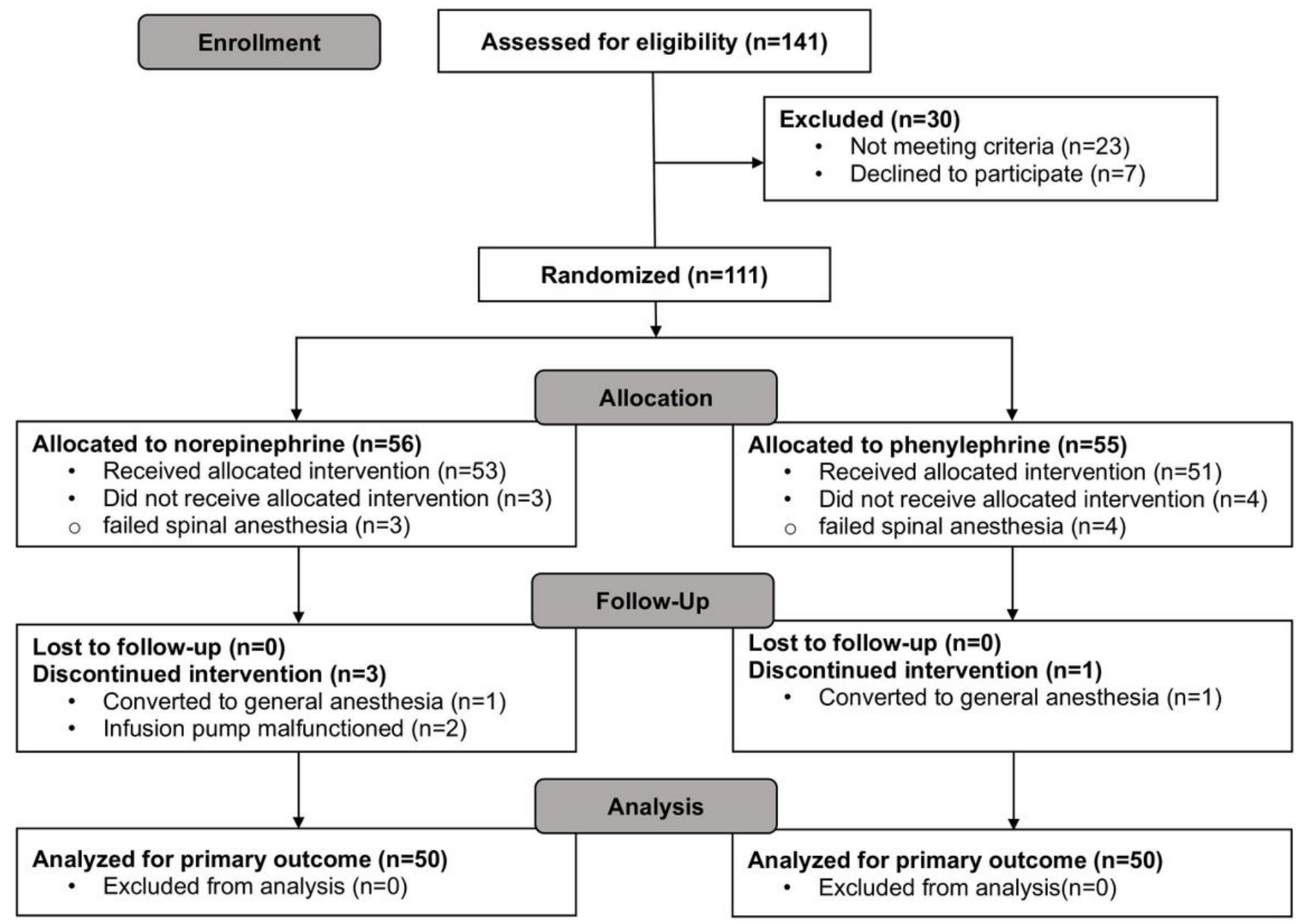

Figure 1

CONSORT flow diagram showing patient recruitment and flow. 


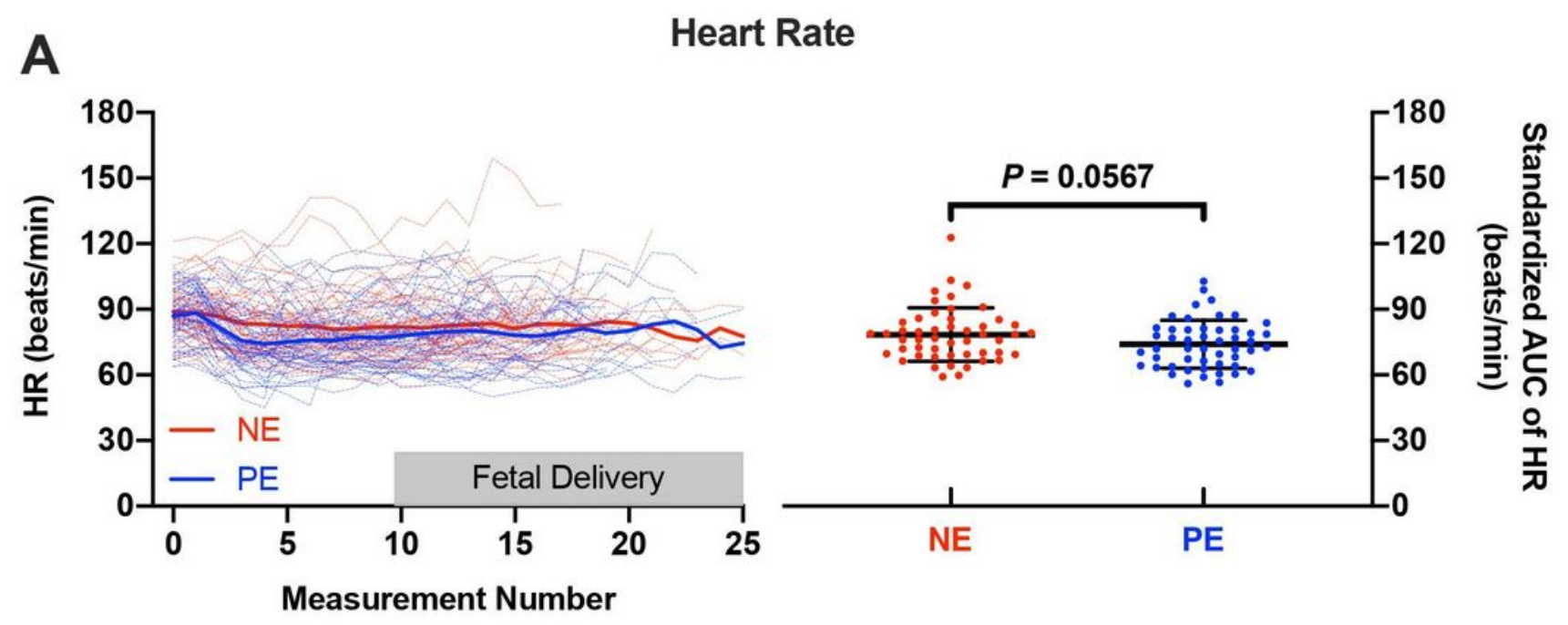

B
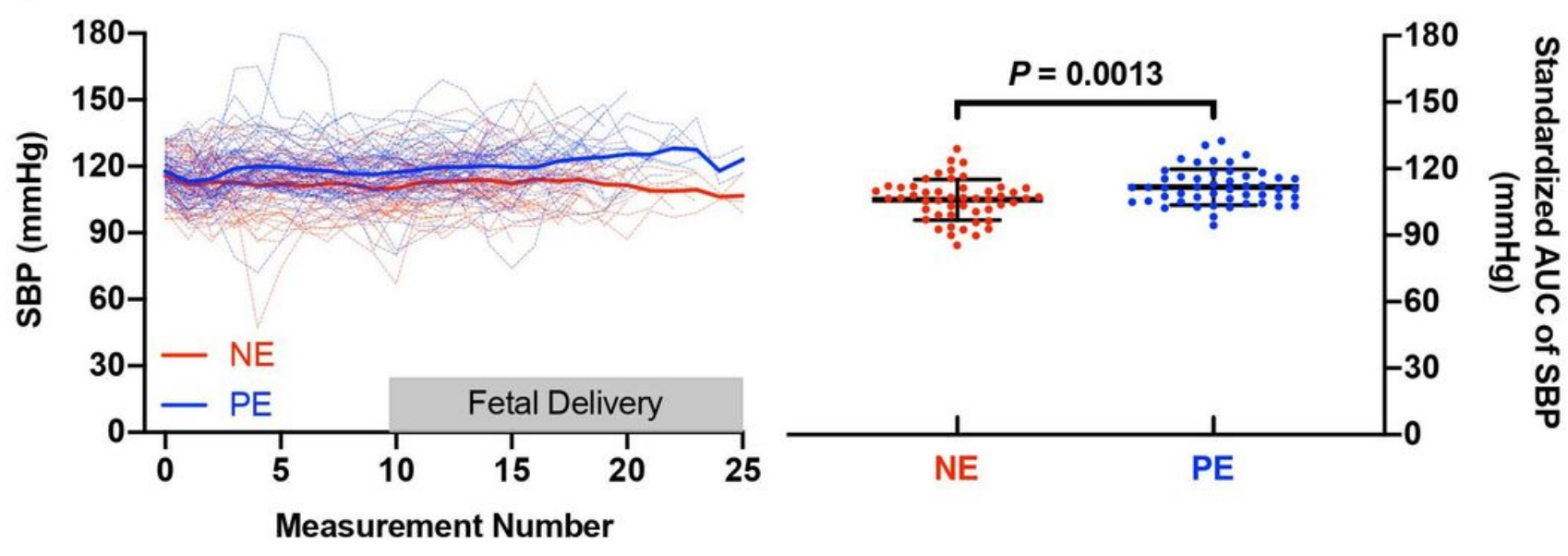

Figure 2

Serial changes in heart rate (A) and systolic blood pressure (B) following norepinephrine (lines in red) or phenylephrine (lines in blue) infusion. Compared with phenylephrine, infusion of norepinephrine is not associated with less decrease in heart rate $(p=0.0567)$ but with a lower systolic blood pressure $(P=$ 0.0013) over the study period. On the left side, dotted lines indicate individual measurements while solid lines indicate the mean values. The shaded area shows the time period within which the newborns are delivered. One the right side, individual data are presented as scattered dots and bars indicate mean and standard deviation. $($ AUC $=$ Area Under the Curve, $H R=$ Heart Rate and SBP $=$ Systolic Blood Pressure $)$. 


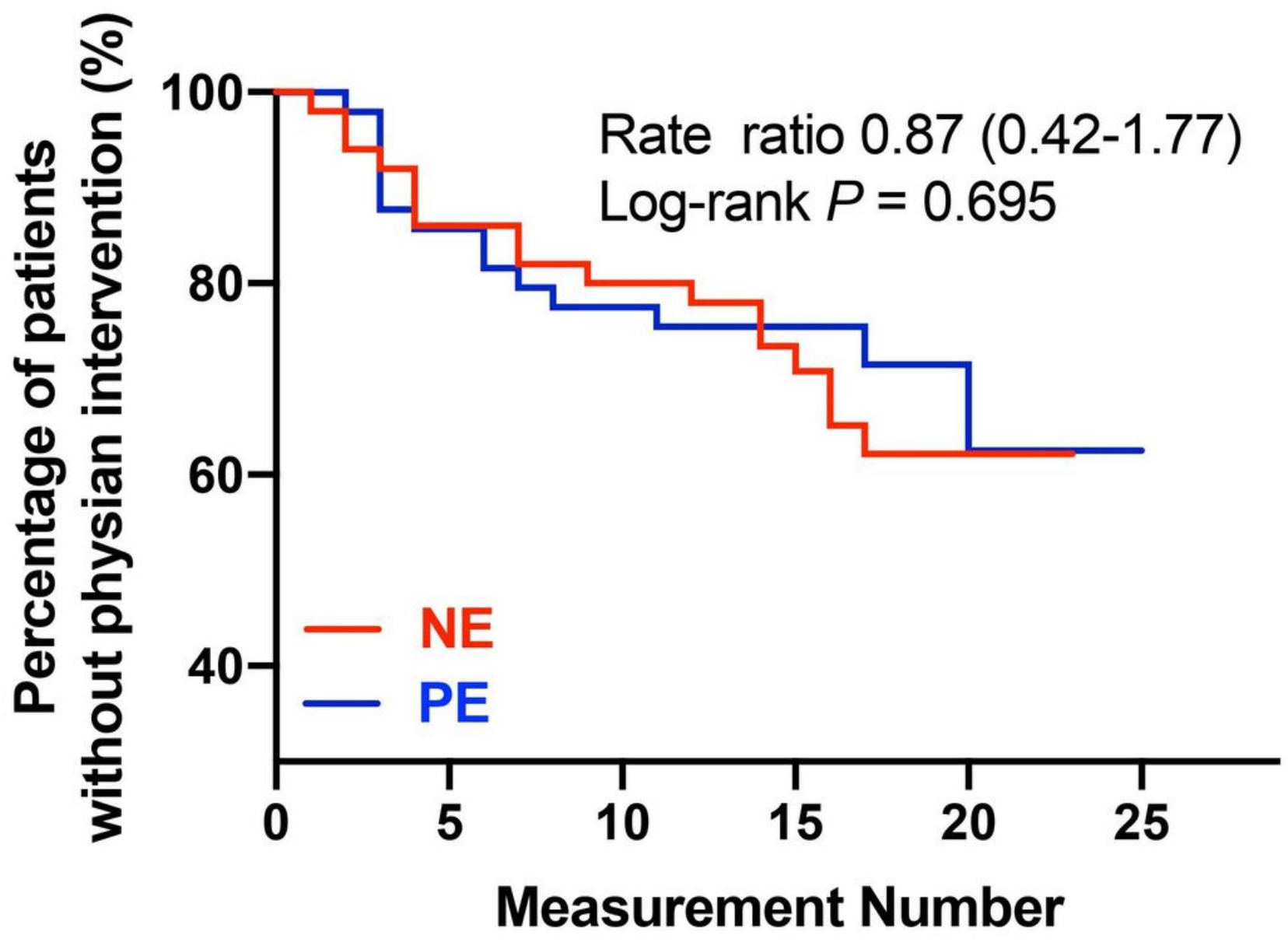

Figure 3

Requirement of physician intervention for correcting maternal hemodynamic abnormalities following norepinephrine or phenylephrine infusion. 